ESAIM: PROCEEDINGS, March 2013, Vol. 39, p. 7-14

M. Belhaq, P. Lafitte and T. Lelièvre Editors

\title{
WAVE PROPAGATION IN EXCITABLE MEDIA THROUGH RANDOMLY DISTRIBUTED HETEROGENEITIES : SIMULATIONS AND COMPARISON TO THE EFFECTIVE MEDIUM THEORY*
}

\author{
SErgio Alonso ${ }^{1}$ And Markus Bär ${ }^{1}$
}

\begin{abstract}
The propagation of traveling waves in excitable media with randomly distributed diffusion coefficient is studied. If the characteristic size of the waves is much larger than the heterogeneity size an effective medium theory based on a self-consistent homogenization approach can be applied. The random distribution of the medium properties is produced by domains of two phases. In each phase the values of the diffusion coefficient are different. The characteristic size of the domains of the two phases is varied in the numerical simulations. The resulting velocities of the traveling waves found by numerical simulations of the random media are compared with the predictions of the effective medium theory. For large size of the heterogeneities in comparison with the diffusion length of the reaction-diffusion system the numerical results show deviations with respect to the predictions.
\end{abstract}

\section{INTRODUCTION}

Nonlinear waves in extended systems can be observed in biological [1] and chemical [2] systems. They result from the interplay of diffusion and nonlinear reaction in systems kept outside the thermodynamic equilibrium [3]. For example, wave propagation of chemical concentrations have been observed in the Belousov-Zhabotinsky reaction [4] and in the catalytic oxidation of $\mathrm{CO}$ on platinum single crystal surfaces [5]. Similar wave phenomena are typical in some biological systems : waves of calcium concentration are observed inside many cells [6], signal waves drive the aggregation processes in populations of collective amoeba Dictyostelium discoideum [7], and traveling action potentials coordinate the regular beat of the heart [8].

While chemical systems are often homogeneous, many biological systems are highly inhomogeneous, and the waves can be strongly disturbed. For example, cardiac tissue is highly inhomogeneous and the effect of randomly distributed dead cells or disconnections among cells have been studied numerically with heterogeneous cardiac models $[9,10]$. The propagation velocity depends on the fraction of heterogeneities in the tissue. Cardiac cell cultures are more inhomogeneous than the tissue, and studies on electric wave propagation in cell cultures show similar properties [11]. Heterogeneous models have been employed to study calcium waves in cells [12].

The homogeneous assumption in chemical systems can be modified by the use of externally imposed inhomogeneities. With this aim the Belousov-Zhabotinsky reaction has been widely employed. The use of masks permits the introduction of patterned illumination into the photosensitive version of the reaction [13]. Heterogeneities have been also achieved by confining chemical species in small compartments : reactions in microemulsion systems [14] and on porous catalytic beads [15]. Another example of heterogeneous chemical pattern formation is the introduction of nonreactive inclusions in the catalytic oxidation of CO on platinum surfaces [16].

\footnotetext{
* This research was supported by the German Science Foundation (DFG) within the framework of SFB 910 "Control of selforganizing nonlinear systems : Theoretical methods and concepts of application"

1 Physikalisch-Technische Bundesanstalt, Abbestrasse 2-12, 10587 Berlin, Germany
} 
In some of the examples shown above the heterogeneities are large compared with the characteristic size of the patterns and an homogenization theory is not possible. However, for the case of small heterogeneities there are general methods to obtain effective reaction rates and diffusion coefficients for randomly heterogeneous reaction-diffusion systems with an arbitrary number of chemical species and linear [17] or nonlinear [18,19] kinetics. The effective medium theory can be also applied to excitable media [20] and it was able to reproduce the main characteristics of the patterns formed in the microemulsion system [21]. Other homogenization theories has been also previously employed for periodically inhomogeneous systems [22].

We consider media supporting wave propagation with random changes in the propagation properties. We apply a homogenization approach to obtain effective diffusion and reaction properties of a random heterogeneous system [17,18] and compare the analytical predictions with the results of numerical simulations in excitable systems [20] and with experiments [21]. Here, we employ a model of excitable media as in [20], however, we restrict the study to $1 \mathrm{D}$ and increase the characteristic size of the heterogeneities to evaluate the limits of the theory, originally developed for small heterogeneities [20].

\section{THEORY}

Chemical species $u$ and $v$ undergo reaction dynamics in the system. Particles of specie $u$ can diffuse throughout the system, however, their reaction rate and diffusion coefficient depend on the position :

$$
\begin{aligned}
\frac{\partial u}{\partial t} & =k(\vec{r}) R_{u}(u, v)+\nabla \cdot(D(\vec{r}) \nabla u) \\
\frac{\partial v}{\partial t} & =R_{v}(u, v) .
\end{aligned}
$$

We consider a binary heterogeneous medium composed of a random distribution of two types of phases, domains of phase 2 are dispersed in a medium of phase 1. The diffusion coefficient and the reaction rate may differ in the different phases. We assume that the diffusion coefficient and the reaction rate inside domains of phase 1 and phase 2 are, respectively, $D_{1}, k_{1}$ and $D_{2}, k_{2}$.

If the characteristic domain size in this heterogeneous system is small compared to the scales of interest, one may describe the reaction-diffusion dynamics in terms of effective medium equations :

$$
\begin{aligned}
\frac{\partial\langle u\rangle}{\partial t} & =k_{e} R_{u}(\langle u\rangle,\langle v\rangle)+\nabla \cdot\left(D_{e} \nabla\langle u\rangle\right) ; \\
\frac{\partial\langle v\rangle}{\partial t} & =R_{v}(\langle u\rangle,\langle v\rangle) ;
\end{aligned}
$$

where the angular brackets correspond to a coarse-graining average over a distance that is large compared to the sizes of the inclusions of phase 2 in phase 1 . The values of the effective diffusion coefficient $D_{e}$ and effective reaction rate $k_{e}$ can be obtained for a particular fraction $\phi$ of phase 2 , see below.

The effective values can be derived under the assumption that the characteristic domain size $\ell$ is small compared to the characteristic pattern scale $\lambda[17-19]$. This means that the predictions of the effective medium theory described above can be compared to numerical results provided the diffusion lengths of both phases, associated to $D_{1}$ and $D_{2}$, are larger than the characteristic length of the heterogeneity.

\subsection{Effective diffusion coefficient}

With a fraction $\phi$ of phase 2 and $(1-\phi)$ of phase 1 and following [19] we can define implicitly an effective diffusion coefficient $D_{e}$ :

$$
(1-\phi) \frac{D_{1}-D_{e}}{D_{1}+(d-1) D_{e}}+\phi \frac{D_{2}-D_{e}}{D_{2}+(d-1) D_{e}}=0
$$




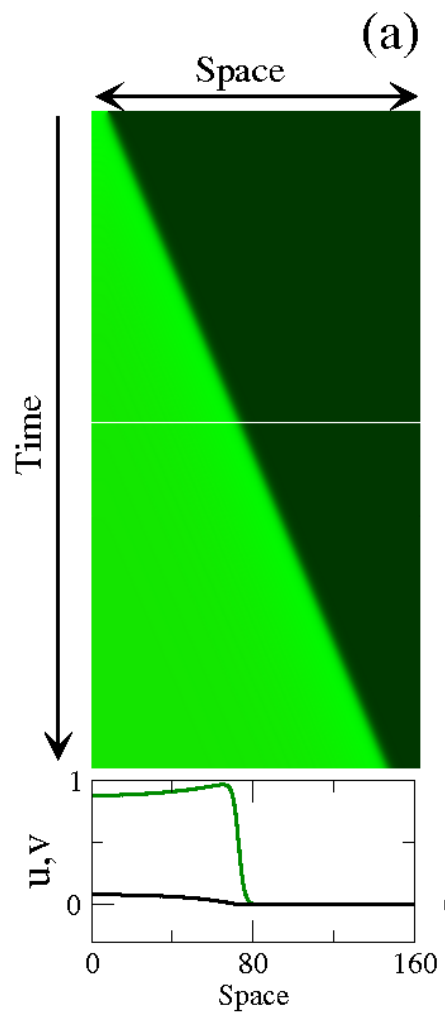

(a)

(b)

(c)
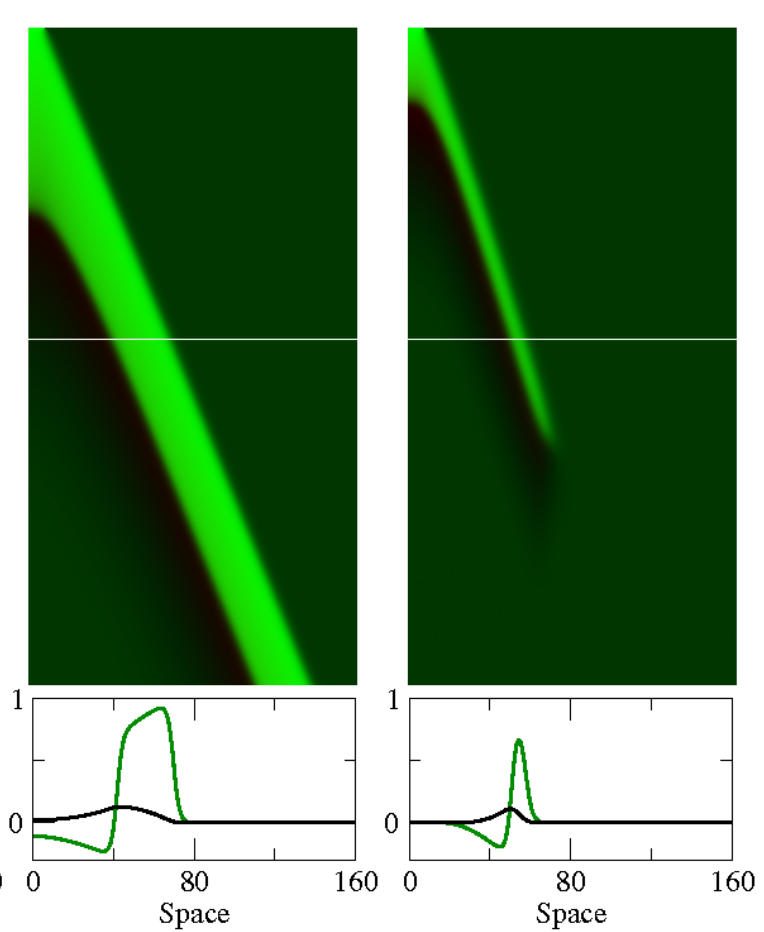

FiguRE 1. Spatio-temporal plots of the evolution of a traveling wave solution of Eqs.(8-9), and the profile of the activator and inhibitor concentrations during a intermediate state (marked with a thin white line in the spatio-temporal plot). Three regimes are shown corresponding to different values of the parameter $b$ : (a) bistable, with $b=0.1$; (b) excitable, with $b=0.25$, and (c) nonexcitable, with $b=0.56$. Simulations are done in a $1 \mathrm{D}$ system $L=160$ during time $T=600$. Discretization values are $: \Delta t=0.002$ and $\Delta x=0.2$.

where the parameter $d$ denotes the spatial dimension of the system, here $d=1$ corresponding to $1 \mathrm{D}$. It is interesting to consider two limits of Eq.(5). If $D_{1} \sim D_{2}$ the effective coefficient predicts a linear change between the two values : $D_{e}(\phi)=D_{1}-\phi\left(D_{1}-D_{2}\right)$. In the opposite case $D_{2} \rightarrow 0$, the theory predicts for $d>1$ a percolation threshold $\phi_{t h}$ which produces $D_{e}\left(\phi_{t h}\right) \sim 0$, for more details see [18].

Equation (5) can be solved to obtain the explicit expression of the effective diffusion coefficient on the fraction of phase 2 for the particular case $d=1$ :

$$
D_{e}^{-1}=D_{2}^{-1} \phi+D_{1}^{-1}(1-\phi)
$$

which corresponds to the weighted harmonic mean of the diffuse coefficients. For $d=1$ the limit $D_{2} \rightarrow 0$ produces $D_{e} \sim 0$ independently of $\phi$.

\subsection{Effective reaction rate}

The effective reaction rate $k_{e}$ is given by the weighted arithmetic mean [19] :

$$
k_{e}(\phi)=(1-\phi) k_{1}+\phi k_{2} .
$$




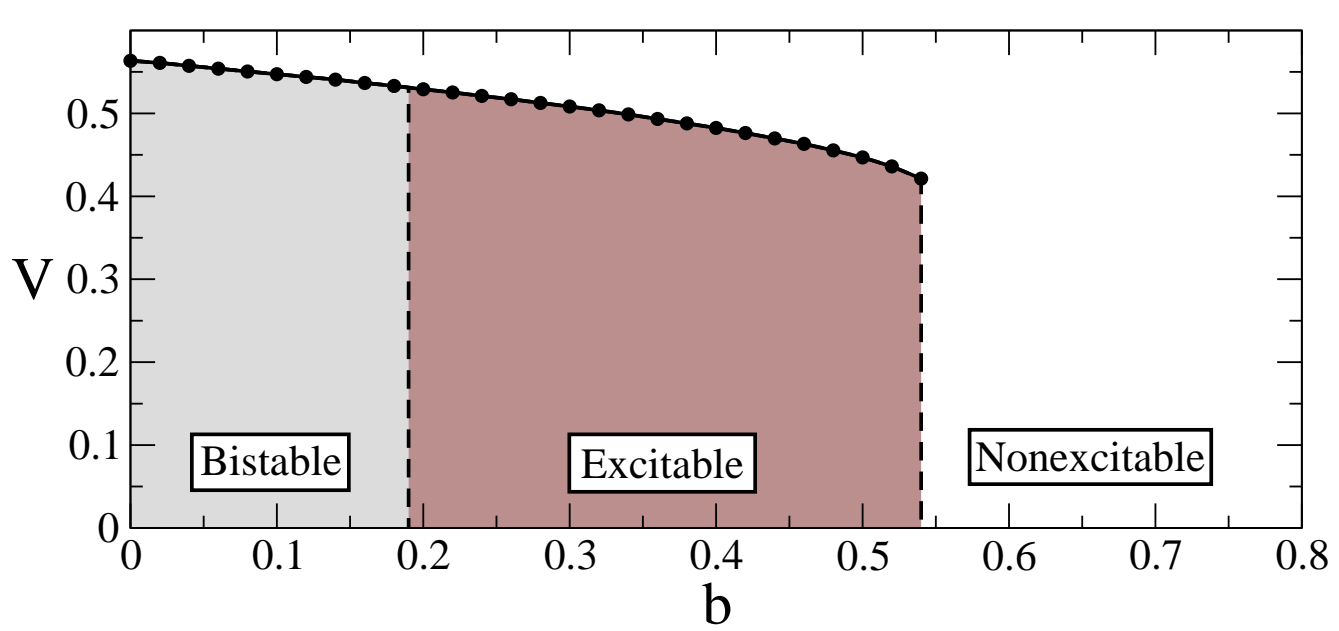

Figure 2. Dependence of the velocity of a traveling wave solution of Eqs.(8-9) on the excitability parameter $b$. The width of the wave is employed to determine the boundary among bistable, excitable and nonexcitable media.

One uses the fact that reaction terms depend on the local concentrations, are uncorrelated and represent the reaction rate in a local mean field approximation, see $[18,19]$. Below, we assume constant reaction rate $k=k_{1}=k_{2}$. The study can be extended to the case of heterogeneous reaction rate using Eq.(7).

\section{MODEL}

\subsection{Reaction-diffusion equations}

We consider a generic activator-inhibitor model of active media :

$$
\begin{aligned}
& \frac{\partial u}{\partial t}=k(u(1-u)(u-a)-v)+\nabla \cdot(D \nabla u) \\
& \frac{\partial v}{\partial t}=\epsilon(b u-v)
\end{aligned}
$$

where $u$ and $v$ represents some activator, respectively, inhibitor. The parameter $D$ is the diffusion coefficient, and $k$ is the reaction rate of the activator (typically we fix the length and time scales keeping $D=1$ and $k=1$ ). The parameter $\epsilon=0.02$ denotes the ratio of the characteristic time scales of activator and inhibitor, which has to be small $\epsilon \ll 1$ to obtain pulses in the excitable regime. Finally, $a$ and $b$ are parameters of the kinetics. We keep the parameter $a=0.1$ and we vary the value of $b$ to obtain different dynamics.

Equations (8-9) are integrated using the explicit Euler method for the temporal evolution and finite differences for the spatial discretization. These equations admit different types of traveling wave solutions, compare Fig. 1. The first example corresponds to a traveling front in a bistable medium. The front produces the transition from the metastable to the stable uniform solution, see Fig. 1(a). The second example shows a traveling pulse in an excitable medium which corresponds to a nonlinear excitation that after a characteristic refractory time recovers to the rest state, see Fig. 1(b). The velocity and the spatial profile of the traveling waves are uniquely defined by the parameters of the system, see Fig. 2.

A bistable and an excitable regime are observed for small respectively large values of $b$. For values of $b$ above a certain value the system becomes subexcitable and there is no pulse propagation, see Fig. 1(c). Wave velocity is calculated from numerical simulations and plotted in Fig. 2. Decreasing the value of the parameter $b$ the 
(a)

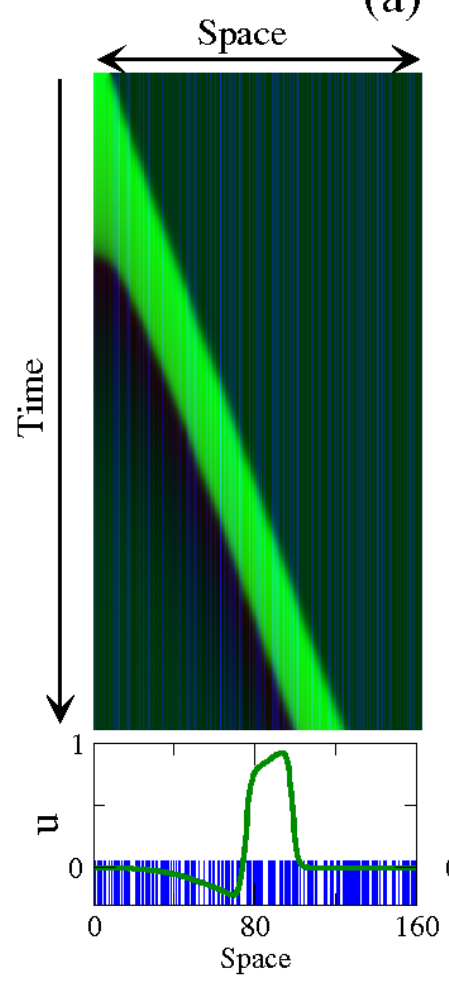

(b)
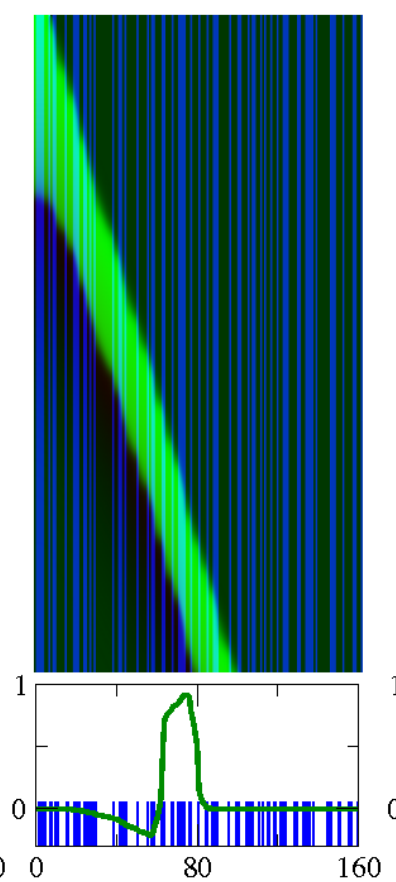

Space (c)
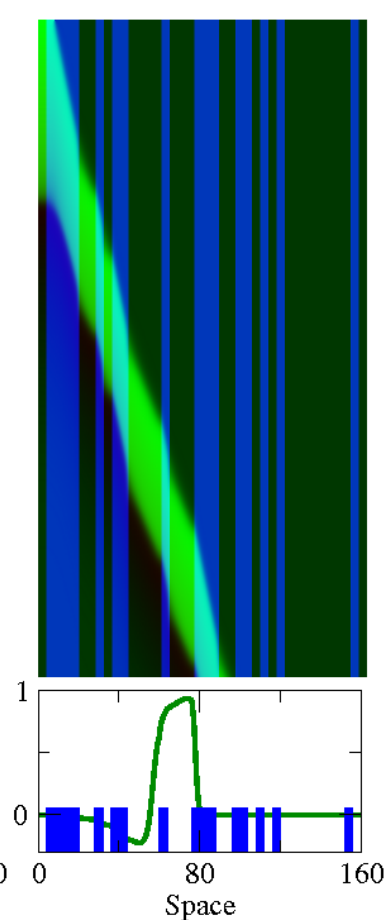

FIGURE 3. Spatio-temporal plots of the traveling wave solution of Eqs.(8-9) under excitable conditions $(b=0.25)$ and the profile of the activator concentration during a intermediate state. Three realizations are shown corresponding to different values of the parameter $: \ell=0.2$ and $\phi=0.2$ (a), $\ell=1$ and $\phi=0.4$, (b) and $\ell=4$ and $\phi=0.4$ (c). Simulations are done in a 1D system $L=160$ during time $T=600$. Discretization values are : $\Delta t=0.002$ and $\Delta x=0.2$.

width of the pulse increases, see Fig. 1(b). For $b<b_{c}$ the system becomes bistable, see Fig. 2, and the front solution is stable, see Fig. 1(a).

In the limits $b \rightarrow 0$ or $\epsilon \rightarrow \infty$, equation Eqs.(9) is irrelevant and the model reduces to a particular version of the Schlögl model [23] given by Eq.(8) with $v=0$. Then, the velocity of the front reads

$$
V_{o}=\sqrt{\frac{D k}{2}}(1-2 a),
$$

which depends on the square root of the diffusion coefficient and reaction rate. From a simple dimensional analysis we keep this dependence for $b>0(V \propto \sqrt{D k})$. Using $D=D_{e}$ and Eq.(6) the wave velocity for $b>0$ reads :

$$
V \propto \sqrt{\frac{D_{1} D_{2} k}{D_{1} \phi+D_{2}(1-\phi)}},
$$




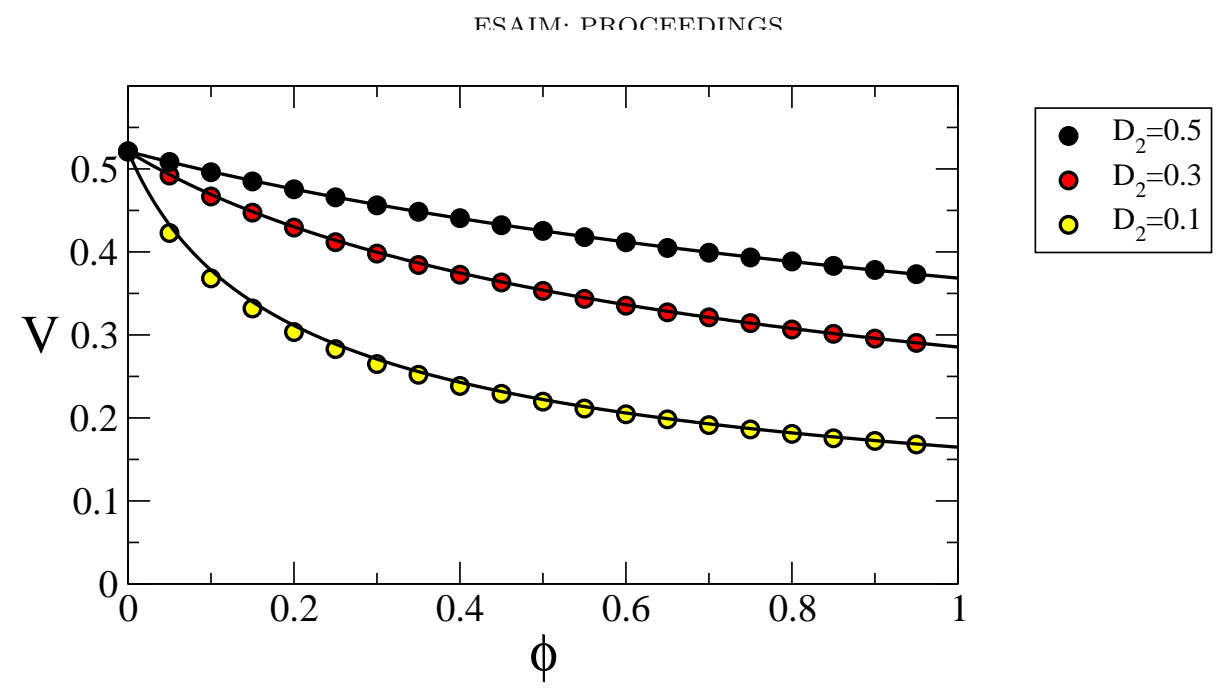

Figure 4. Dependence of the velocity of a traveling wave solution of Eqs.(8-9) on the fraction $\phi$ of phase 2 , for different values of the diffusion coefficient in phase $2\left(D_{1}=1\right)$. Points correspond to the average of ten realizations and lines are the predictions of the effective medium theory Eq.(11). Simulations are done in a $1 \mathrm{D}$ system $L=320$ during the time necessary to cross the whole system. The characteristic size of the domains are $\ell=0.2$. Discretization values are : $\Delta t=0.002$ and $\Delta x=0.2$.

where the proportionality constant is obtained from a numerical simulation of the case $\phi=0$. The characteristic pattern scale corresponds to the front width, which can be estimated from the parameter values by

$$
\lambda=4 \sqrt{\frac{2 D_{1}}{k}}
$$

which, for the values of the parameters employed here, corresponds to $\lambda=5.5$. We consider the propagation of waves in excitable media $(b=0.25)$, we vary the fraction $\phi$ and keep the model parameters constant.

\subsection{Random heterogeneities}

The continuous medium described by Eqs. (8-9) is replaced by a discrete grid where each site is connected by coupling to the nearest neighbors. We employ the parameter $D(\vec{r})$ to introduce the heterogeneities in the numerical simulations $[18,19]$. Heterogeneous diffusion is introduced by spatially random binary distribution $\left(D_{1}\right.$ and $D_{2}$ ) of the value $D$ between each couple of nodes. We associate the phase 1 , corresponding to $D_{1}=1$, to the unperturbed phase and the phase 2 to the abnormal phase forming the heterogeneities. This allows to define the fraction of heterogeneities $\phi$ employed in the numerical simulations.

The flux $(\vec{J}=-D \vec{\nabla} c$ in the continuum description) between two nodes of the grid can be calculated in the discrete description from $J_{i j}=-D_{i j}\left(c_{i}-c_{j}\right) / \Delta x$, where $D_{i j}$ corresponds to the diffusion between the nodes $i$ and $j$. The value of the diffusion coefficient $D_{i j}$ can take only two different values $D_{1}$ and $D_{2}$. We randomly choose the values of $D_{i j}$ as in [20]. However, we keep the value of $D_{i j}$ constant along a distance $\ell$ for the introduction of a characteristic size of the heterogeneities.

\section{Results}

We study the dynamics of traveling waves in randomly inhomogeneous excitable media. We vary the fraction and size of the heterogeneites, i.e. fraction of phase 2. The heterogeneities are introduced in the diffusion coefficient. Typical examples of the dynamics observed in the simulations are shown in the spatio-temporal 

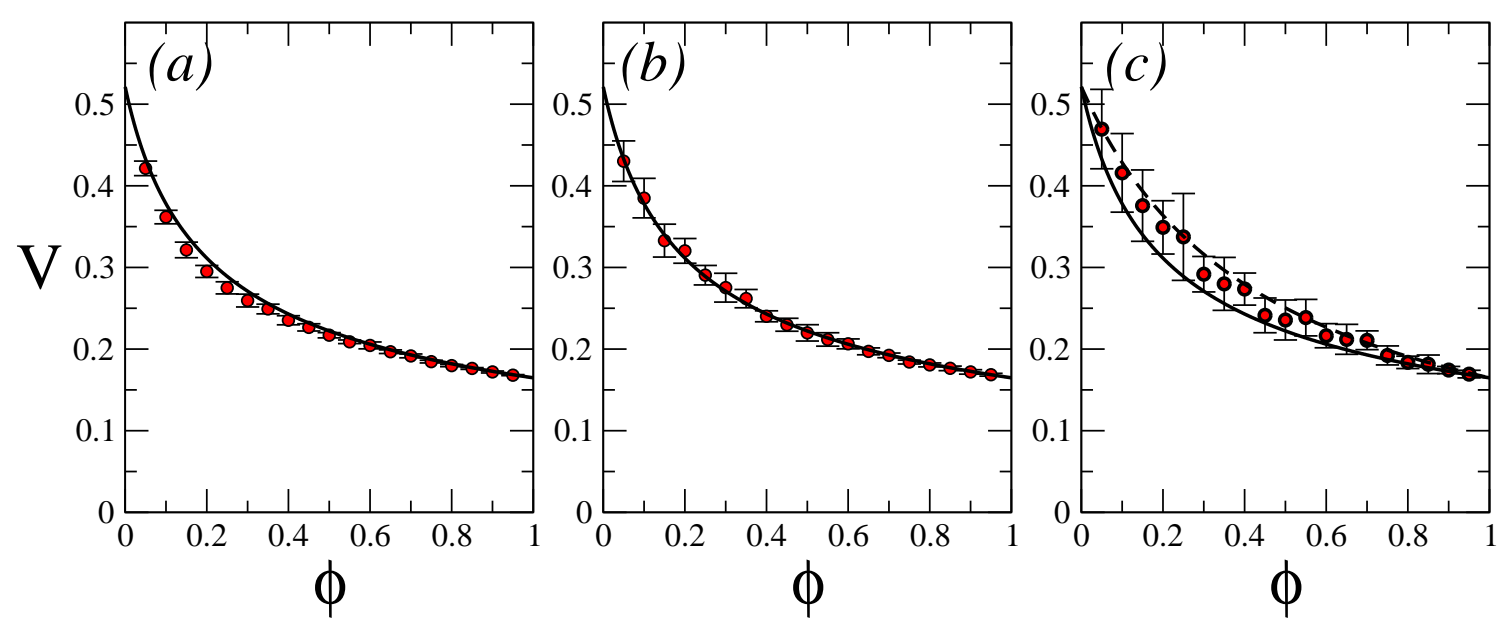

FIGURE 5. Dependence of the velocity of a traveling wave solution of Eqs.(8-9) on the fraction $\phi$ of phase 2 for different sizes of the domains : $\ell=0.6$ (a) $\ell=2$ (b) and $\ell=10$ (c). Points correspond to the average of ten realizations, solid lines are the predictions of the effective medium theory Eq.(11) and dashed line the prediction for large domains Eq.(13). Simulations values and conditions as in Fig.4.

plots on Fig.3. Waves propagate almost homogeneously if the size of the heterogeneities is small, see Fig.3(a), and they propagate less homogeneously if the size is large, see Fig.3(c). For comparison, the relative thickness of the heterogeneities is shown.

To study the dependence of the velocity of the waves on the fraction of the heterogeneities we consider two cases depending on the comparison between the size of the heterogeneities $(\ell)$ and the characteristic pattern size $(\lambda)$ : small $(\ell<\lambda)$ and large $(\ell>\lambda)$ heterogeneities.

\subsection{Small heterogeneities}

In Fig.4, numerical results in 1D are compared with the predictions of the effective medium theory based on Eq. (11). The heterogeneities are introduced in the discretization level. The theory reproduces the numerical results for large values of the diffusion coefficient in the second phase, i.e. similar values of the diffusion coefficients in both phases. However, decreasing the diffusion coefficient we reduce the length of the front and the theory fails when the diffusion coefficients are very different $\left(D_{2} / D_{1}<0.05\right)$ and the characteristic length of the front is similar to the size of the heterogeneities. However simulations shown in Fig. 4 are in good agreement with the theory.

Numerical simulations in two [18] and three [19] dimensions show the same agreement with the predictions of the effective medium theory for similar reaction-diffusion systems.

\subsection{Large heterogeneities}

There is a window of sizes of the heterogeneities $(0<\ell<\lambda)$ where the numerical simulations confirm the predictions of the effective medium theory, see Fig.5(a-b). However, for large heterogeneities the effective medium theory fails to describe the wave dynamics. For $\ell>\lambda$ the wave propagates through large domains and the average velocity is derived assuming stationary dynamics in each domain. The time required to cross a distance $L$ is $t=L V^{-1}$ and corresponds to the sum of the times employed in phase $1\left(t_{1}=(1-\phi) L V_{1}^{-1}\right)$ and in phase $2\left(t_{2}=\phi L V_{2}^{-1}\right)$. Thus, the average velocity corresponds to the weighted harmonic mean of the 
velocities $V^{-1}=V_{2}^{-1} \phi+V_{1}^{-1}(1-\phi)$, see [13]. Finally, assuming $V_{1} \propto \sqrt{D_{1} k}$ and $V_{2} \propto \sqrt{D_{2} k}$, one obtains :

$$
V \propto \frac{\sqrt{D_{1} D_{2}} \sqrt{k}}{\sqrt{D_{1}} \phi+\sqrt{D_{2}}(1-\phi)},
$$

with a different dependence on $\phi$, compared with Eq.(11). In Fig.5(c) this curve is plotted for large domains and the averaged velocity is well reproduced by this expression. Note also the increase of the dispersion on the mean value of the velocity, error bars in Fig.5, with the size of the heterogeneities.

\section{Conclusions}

We have compared the velocity of traveling waves obtained by numerical simulations in heterogeneous excitable media with the predictions of an effective medium theory. The effective medium theory for reactiondiffusion systems predicts the value of the effective diffusion coefficient, which can be calculated from Eq. (5). This quantity permits the calculation of an effective velocity of the traveling waves which can be directly compared with the traveling wave obtained by numerical simulations in heterogeneous excitable media. The comparison of the effective medium theory with simulations shows a good agreement for large diffusion coefficients $D_{1}$ and $D_{2}$ and small size of the domain of phases 1 and 2. To calculate the velocity we need the effective diffusion coefficient obtained from the weighted harmonic mean of the diffusion coefficients. For large domains the numerical results differ from the effective medium theory predictions and the average velocity of the traveling wave approaches the weighted harmonic mean of the velocities in both phases.

\section{REFERENCES}

[1] J. P. Keener and J. Sneyd, Mathematical Physiology (Springer, New York, 1998).

[2] R. Kapral and K. Showalter (Eds.), Chemical Waves and Patterns (Kluwer, Dordrecht, 1994).

[3] R. C. Desai and R. Kapral, Dynamics of Self-Organized and Self-Assembled Structures (Cambridge University, Cambridge, England, 2009).

[4] A. N. Zaikin and A. M. Zhabotinskii, Nature (London) 225, 535 (1970).

[5] S. Jakubith, H. H. Rotermund, W. Engel, A. von Oertzen, and G. Ertl, Phys. Rev. Lett. 65, 3013 (1990).

[6] J Lechleiter, S Girard, E Peralta and D Clapham Science 252, 123-126 (1991).

[7] F. Siegert and C. J. Weijer, Physica D 49, 224 (1991).

[8] D. P. Zipes and J. Jalife Cardiac Electrophysiology : From Cell to Bedside (Philadelphia, PA : Saunders, 2004)

[9] K. H. W. J. Ten Tusscher and A. V. Panfilov, Phys. Rev. E 68, 062902 (2003).

[10] S. Alonso, M. Bär and A. V. Panfilov, Chaos 21, 013118 (2011).

[11] B. E. Steinberg, L. Glass, A. Shrier, and G. Bub, Philos. Trans. R. Soc. London, Ser. A 364, 1299 (2006).

[12] R. Thul and M. Falcke, Phys. Rev. Lett. 93, 188103 (2004).

[13] I. Sendiña-Nadal, A. P. Muñuzuri, D. Vives, V. Pérez-Muñuzuri, J. Casademunt, L. Ramírez-Piscina, J. M. Sancho, and F. Sagués, Phys. Rev. Lett. 80, 5437 (1998).

[14] V. K. Vanag and I. R. Epstein, Phys. Rev. Lett. 87, 228301 (2001).

[15] A. F. Taylor, M. R. Tinsley, F. Wang, Z. Huang, and K. Showalter, Science 323, 614 (2009).

[16] M. Bär, A. K. Bangia, I. G. Kevrekidis, G. Haas, H.-H. Rotermund, and G. Ertl, J. Phys. Chem. 100, 19106 (1996).

[17] D. Bedeaux and R. Kapral, J. Chem. Phys. 79, 1783 (1983).

[18] S. Alonso, R. Kapral and M. Bär, Phys. Rev. Lett. 102, 238302 (2009).

[19] S. Alonso, M. Bär and R. Kapral, J. Chem. Phys. 131, 214102 (2009).

[20] S. Alonso, J. Löber, M. Bär, and H. Engel, Eur. Phys. J. Special Topics 187, 31-40 (2010).

[21] S. Alonso, K. John and M. Bär J. Chem. Phys. 134, 094117 (2011).

[22] J.P. Keener, Physica D, 136, 1 (2000).

[23] F. Schlögl, Zeitschrift für Physik A Hadrons and Nuclei, 253, (1972) 147. 